\title{
Effect of Single-Nucleotide Polymorphisms on Decline of Dopamine Transporter Availability in Parkinson's Disease
}

\author{
Seunghyeon Shin ${ }^{a}$ \\ Keunyoung Kim ${ }^{\mathrm{a}}$ \\ Jae Meen Lee ${ }^{b}$ \\ Eun-Joo Kim ${ }^{\mathrm{C}}$ \\ Seong-Jang Kim ${ }^{\mathrm{d}}$ \\ In Joo Kim \\ Kyoungjune Pak ${ }^{\mathrm{a}}$ \\ Myung Jun Lee \\ aDepartments of Nuclear Medicine,
beurosurgery, and 'Neurology,
Biomedical Research Institute,
Pusan National University Hospital,
Busan, Korea
dDepartment of Nuclear Medicine and
Research Institute for Convergence
of Biomedical Science and Technology,
Pusan National University
Yangsan Hospital, Yangsan, Korea
}

Received July 16, 2018

Revised September 24, 2018

Accepted September 28, 2018

\section{Correspondence}

In Joo Kim, MD

Department of Nuclear Medicine,

Biomedical Research Institute,

Pusan National University Hospital,

179 Gudeok-ro, Seo-gu,

Busan 49241, Korea

Tel +82-51-240-7389

Fax +82-51-240-7442

E-mail injkim@pusan.ac.kr

Kyoungjune Pak, MD

Department of Nuclear Medicine, Biomedical Research Institute,

Pusan National University Hospital,

179 Gudeok-ro, Seo-gu,

Busan 49241, Korea

Tel +82-51-240-7389

Fax +82-51-240-7442

E-mail ilikechopin@me.com

Myung Jun Lee, MD

Department of Neurology,

Biomedical Research Institute,

Pusan National University Hospital,

179 Gudeok-ro, Seo-gu,

Busan 49241, Korea

Tel $+82-51-240-7317$

Fax +82-51-240-2783

E-mail mslayer9@gmail.com
Background and Purpose We aimed to determine the association between the annual changes in dopamine transporter (DAT) availability as measured by ${ }^{123} \mathrm{I}$-ioflupane $\left({ }^{123} \mathrm{I}\right.$-FP-CIT) single-photon-emission computed tomography and single-nucleotide polymorphisms (SNPs) known to be risk factors in Parkinson's disease (PD).

Methods In total, 150 PD patients were included from the Parkinson's Progression Markers Initiative database. Specific SNPs that are associated with PD were selected for genotyping. SNPs that were not in Hardy-Weinberg equilibrium or whose minor allele frequency was less than 0.05 were excluded. Twenty-three SNPs met the inclusion criteria for this study. The Kruskal-Wallis test was used to compare annual percentage changes in DAT availability for three subgroups of SNP.

Results None of the 23 SNPs exerted a statistically significant effect $(p<0.0022)$ on the decline of DAT availability in PD patients. However, we observed trends of association $(p<0.05)$ between three SNPs of two genes with the annual percentage change in DAT availability: 1) rs199347 on the putamen $(p=0.0138), 2)$ rs356181 on the caudate nucleus ( $p=0.0105)$, and 3 ) rs3910105 on the caudate nucleus $(p=0.0374)$. A post-hoc analysis revealed that DAT availability was reduced the most for 1 ) the putamen in the CC genotype of rs199347 (vs. CT, $p=0.0199$; vs. TT, $p=$ $0.0164), 2$ ) the caudate nucleus in the TT genotype of rs356181 (vs. CC, $p=0.0081$ ), and 3 ) the caudate nucleus in the CC genotype of rs3910105 (vs. TT, $p=0.0317$ ).

Conclusions Significant trends in the associations between three SNPs and decline of DAT availability in PD patients have been discovered.

Key Words single photon emission computed tomography, ${ }^{123} \mathrm{I}$-ioflupane dopamine transporter, single-nucleotide polymorphism, Parkinson's disease.

\section{INTRODUCTION}

Parkinson's disease (PD), which is characterized by motor symptoms such as rigidity, hypokinesia, and tremor, is a neurological disorder caused by the loss of dopaminergic neurons in the substantia nigra that results in a decreased dopamine level in the striatum. ${ }^{1}$ Moreover, the dopamine transporter (DAT), which is present on the presynaptic dopaminergic nerve terminal and controls dopamine level via the reuptake of dopamine in the synaptic cleft, is decreased with dopaminergic neurodegeneration of nigrostriatal neurons. ${ }^{2,3}$ Since the uptake of ${ }^{123} \mathrm{I}$-ioflupane $\left({ }^{123} \mathrm{I}\right.$-FP-CIT) in the striatum is correlated with DAT density, ${ }^{123}$ I-FP-CIT can be used to differentiate Parkinsonian syndromes with and without presynaptic dopaminergic loss. ${ }^{3,4}$ A systematic review found that DAT imaging had a sensitivity of $100 \%$ in patients with a clinical or neuropathological diagnosis of PD. ${ }^{5}$ It is well known that

(c) This is an Open Access article distributed under the terms of the Creative Commons Attribution Non-Commercial License (https://creativecommons.org/licenses/by-nc/4.0) which permits unrestricted non-commercial use, distribution, and reproduction in any medium, provided the original work is properly cited. 
the DAT uptake of the striatum as measured by DAT imaging is correlated with the severity and duration of PD. ${ }^{6,7}$ The annual reduction rate of striatal DAT uptake is between $6 \%$ and $13 \%$ in $\mathrm{PD}^{7}$

PD was previously considered a nongenetic disease caused by environmental factors such as viral infection or neurotoxins. ${ }^{8}$ However, following Polymeropoulos suggesting a genetic component of PD in 1997, various studies were conducted to identify the genetic factors affecting the etiology of PD using whole-exome sequencing and genome-wide association studies (GWAS) ${ }^{8}$ Mutations causing PD have now been identified in 15 genes, including SNCA, LRRK2, and MAPT. ${ }^{8}$

Few studies have evaluated the correlation between genetic mutations and features of DAT imaging in PD patients. McNeill et al. ${ }^{9}$ used ${ }^{123}$ I-FP-CIT single-photon-emission computed tomography (SPECT) to reveal that a striatal asymmetry index was higher in PD patients with GBA or LRRK2 than in those with PARKIN, PINK2, and SNCA. Huertas et al. ${ }^{10}$ reported that the striatal DAT availability as measured by ${ }^{123}$ I-FP-CIT SPECT in PD patients was associated with SNCA rs356219 and deleterious variants in GBA. However, to the best of our knowledge, no studies have evaluated the correlation between genetic mutations known to be risk factors in PD and chronological changes in striatal DAT availability in PD patients. The present study aimed to determine the association between the annual changes in DAT availability and single-nucleotide polymorphisms (SNPs) known to be risk factors in $\mathrm{PD}$, in order to identify the prognostic value of genetic mutations in $\mathrm{PD}$ patients.

\section{METHODS}

\section{Subjects}

The data used in this study were obtained from the Parkinson's Progression Markers Initiative (PPMI) database (www. ppmi-info.org/data); up-to-date information about the study is available at www.ppmi-info.org. ${ }^{11}$ Males and females aged 30 years or older at screening were included. At baseline, patients with a PD diagnosis within the past 2 years, a Hoehn and Yahr stage not higher than II, a striatal dopamine deficit in ${ }^{123}$ I-FP-CIT SPECT, and who had not received treatment were included according to the PPMI criteria of PD groups. The following exclusion criteria are applied to the PPMI database: a clinical diagnosis of dementia, taking drugs within the previous 6 months that might interfere with ${ }^{123}$ I-FPCIT SPECT, currently taking anticoagulants, having any other medical or psychiatric condition or laboratory abnormality, having taken investigational drugs or devices within 60 days prior to baseline, or showing evidence of clinically significant neurological disorders in magnetic resonance imaging. This study included PD patients who received two ${ }^{123}$ I-FP-CIT SPECT scans at both the initial screening and 12-month follow-up. The medical history, genotyping results, and ${ }^{123} \mathrm{I}$-FP-CIT SPECT scans were downloaded. The PPMI study was approved by the local Institutional Review Boards of all participating sites: Institute for Neurodegenerative Disorders, University of Pennsylvania, University of California, Los Angeles, Coriell Institute for Medical Research, Clinical Trials Coordination Center, Laboratory of Neurogenetics, National Institute on Aging National Institutes of Health, Institute for Neurodegenerative Disorders, Clinical Trials Statistical and Data Management Center, University of Iowa. Written informed consent was obtained from each subject at the time of enrollment for imaging data and clinical questionnaires. All of the study methods were performed in accordance with the relevant guidelines and regulations.

\section{${ }^{123}$ I-FP-CIT SPECT and image analysis}

${ }^{123} \mathrm{I}$-FP-CIT SPECT was performed during the screening visit for all subjects. SPECT scans were acquired $4.0 \pm 0.5 \mathrm{hr}$ (mean \pm SD) after injecting $111-185 \mathrm{MBq}{ }^{123}$ I-FP-CIT. Subjects were pretreated with iodine solution or perchlorate prior to the injection in order to block uptake by the thyroid. Raw data were acquired into a $128 \times 128$ matrix, in steps of 3 or 4 degrees for the total projections. Raw projection data were reconstructed using iterative ordered subset expectation maximization with HERMES software (Hermes Medical Solutions, Stockholm, Sweden). The reconstructed images were transferred to PMOD software (PMOD Technologies, Zürich, Switzerland) for subsequent processing, including attenuation correction.

Downloaded scans were loaded using PMOD (version 3.6, PMOD Technologies) with the ${ }^{123}$ I-FP-CIT template. ${ }^{12}$ The specific binding of ${ }^{123}$ I-FP-CIT regarding the DAT was calculated using a region-of-interest analysis. Standard sets of a volume of interest (VOI) defining the caudate nucleus and putamen based on the Automated Anatomical Labeling atlas $^{13}$ were used. The cerebellum was chosen as a reference region. The VOI template was applied to calculate specific binding ratios (SBRs) of the caudate nucleus and putamen as (target-cerebellum)/cerebellum. The annual percentage change in DAT availability was calculated as [(SBR at 12month follow-up)-(SBR at screening)]/(SBR at screening) $\times$ 100/[(interval between 12-month follow-up and screening, in days)/365].

\section{Genotyping}

All samples were genotyped using the Illumina NeuroX array following the manufacturer's protocol (Illumina, San Di- 
ego, CA, USA). The Genotyping Analysis Module within Genome Studio (version 1.9.4; https://support.illumina.com) was used to analyze data. The genotype frequencies of the SNPs were in Hardy-Weinberg equilibrium (>0.05). SNPs were removed if the minor allele frequency was less than 0.05 . Twenty-three SNPs met the inclusion criteria for this study.

\section{Clinical measurements}

To compare clinical features according to three subgroups of SNP, we used the results of the Movement-Disorder-Society-sponsored Unified Parkinson's Disease Rating Scale (MDSUPDRS) parts II and III. The MDS-UPDRS score was obtained at the time of screening.

\section{Statistical analysis}

Normality was examined using D'Agostino-Pearson omnibus test. The paired $t$-test and the Wilcoxon test were used to compare differences between SBRs at screening and at the 12-month follow-up and in the annual percentage changes in the caudate nucleus and putamen. The Kruskal-Wallis test was used to compare the annual percentage changes in DAT availability for three subgroups of SNP. Since 23 SNPs met the inclusion criteria for this study, significance was set at $\alpha=$ $0.05 / 23(0.0022)$ to correct for multiple comparisons. Posthoc analysis was performed for pairwise comparisons of subgroups using Dunn's test. The Kruskal-Wallis test was used to analyze the difference of the MDS-UPDRS score according to three subgroups of SNPs. Statistical analyses were performed using GraphPad Prism 7 for Mac OS X (GraphPad Software, San Diego, CA, USA), and MedCalc software package (version 12.6.0.0, MedCalc, Ostend, Belgium).

\section{RESULTS}

This study included 150 PD patients (101 males and $49 \mathrm{fe}-$ males). The genotype and allele frequencies of the 23 SNPs in the PPMI database that met the inclusion criteria for this study are listed in Table 1 . The SBRs of the caudate nucleus and putamen were $1.21 \pm 0.30$ and $1.18 \pm 0.28$, respectively, at screening, and $1.12 \pm 0.27$ and $1.08 \pm 0.27$ at the 12 -month follow-up, thereby showing significant reductions for both structures (both $p<0.0001$ ) (Fig. 1). The mean interval between screening and the 12-month follow-up was 391.3 days. The annual percentage reduction in DAT availability was greater for the putamen $(7.18 \%)$ than for the caudate nucleus $(5.27 \%)(p=0.0417)$. The characteristics of the included subjects are summarized in Table 2.

None of the 23 SNPs exerted a statistically significant effect $(p<0.0022)$ on the decline of DAT availability in PD patients. However, we observed trends of association $(p<0.05)$
Table 1. Genotype frequencies for alleles

\begin{tabular}{|c|c|c|c|c|}
\hline SNP & & enotype ( & & MAF \\
\hline rs10797576 & CC (112) & СТ (36) & $\Pi(2)$ & 0.13 \\
\hline rs11060180 & $\mathrm{AA}(50)$ & $A G(69)$ & GG (31) & 0.44 \\
\hline rs11158026 & CC (63) & CT (75) & $\Pi(12)$ & 0.33 \\
\hline rs115462410 & CC (128) & CT (21) & $\Pi(1)$ & 0.08 \\
\hline rs11724635 & AA (53) & $\mathrm{AC}(65)$ & CC (32) & 0.43 \\
\hline rs11868035 & AA (13) & $A G(61)$ & GG (76) & 0.34 \\
\hline rs12456492 & AA (71) & $A G(67)$ & GG (12) & 0.30 \\
\hline rs12637471 & AA (5) & $A G(49)$ & GG (96) & 0.20 \\
\hline rs14235 & AA (25) & $A G(75)$ & GG (49) & 0.42 \\
\hline rs17649553 & CC (103) & CT (43) & $\Pi(4)$ & 0.17 \\
\hline rs1955337 & GG (109) & GT (38) & $\Pi(3)$ & 0.15 \\
\hline rs199347 & CC (20) & CT (74) & $\Pi(56)$ & 0.38 \\
\hline rs2414739 & AA (81) & $A G(60)$ & GG (9) & 0.26 \\
\hline rs329648 & CC (62) & CT (62) & $\Pi(26)$ & 0.38 \\
\hline rs34311866 & AA (85) & AG (53) & GG (12) & 0.26 \\
\hline rs356181 & CC (43) & CТ (75) & $\Pi(32)$ & 0.46 \\
\hline rs3910105 & CC (29) & CT (74) & $\Pi(47)$ & 0.44 \\
\hline rs55785911 & AA (22) & AG (73) & GG (55) & 0.39 \\
\hline rs591323 & AA (12) & AG (65) & GG (73) & 0.30 \\
\hline rs6430538 & CC (46) & CT (68) & $\Pi(36)$ & 0.47 \\
\hline rs6812193 & CC (64) & CT (69) & $\Pi(17)$ & 0.34 \\
\hline rs76904798 & CC (106) & CT (43) & $\Pi(1)$ & 0.15 \\
\hline rs823118 & CC (26) & CT (81) & $\Pi(43)$ & 0.44 \\
\hline
\end{tabular}

MAF: minor allele frequency, SNP: single-nucleotide polymorphism.

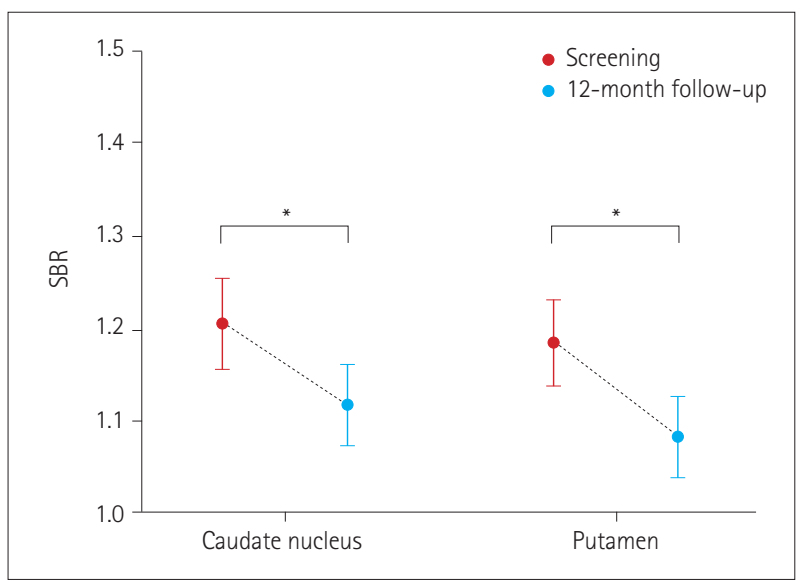

Fig. 1. Serial changes in the SBR between screening and 12-month follow-up in both the caudate nucleus and putamen ${ }^{*} p<0.0001$; data are means with $95 \% \mathrm{Cls}$ ). SBR: specific binding ratio.

between three SNPs of two genes in terms of the annual percentage changes in DAT availability: 1) rs199347 on the putamen $(p=0.0138), 2)$ rs356181 on the caudate nucleus ( $p=$ $0.0105)$, and 3) rs3910105 on the caudate nucleus ( $p=0.0374)$. A post-hoc analysis revealed that DAT availability was reduced the most for 1) the putamen in the CC genotype of 
rs199347 (vs. CT, $p=0.0199$; vs. TT, $p=0.0164$ ), 2) the caudate nucleus in the TT genotype of rs356181 (vs. CC, $p=0.0081$ ), and 3) the caudate nucleus in the CC genotype of rs3910105 (vs. TT, $p=0.0317$ ) (Fig. 2). However, the MDS-UPDRS score did not differ between the rs199347, rs356181, and rs3910105 SNPs.

\section{DISCUSSION}

SNPs are variations in the positions of single nucleotides, and they have a frequency of at least $1 \%$ in the human genome. ${ }^{14}$ An SNP can exert its affects at the transcriptional, translational, and posttranslational levels to result in alterations to gene expression and protein stability. ${ }^{15}$ GWAS have identified numerous SNPs associated with cancer, infection, diabetes, and autoimmune, cardiovascular, gastrointestinal, and neuropsychiatric diseases. ${ }^{15}$ Polymorphisms in the SLC6A3 gene encoding DAT have been associated with DAT availability, and the number of tandem 9-repeats in the 3 ' untrans-

Table 2. Characteristics of the study subjects

\begin{tabular}{lc}
\hline \multicolumn{1}{c}{ Characteristic } & Value \\
\hline Age (years) & $63.1 \pm 9.3$ \\
Sex (male/female) & $101 / 49$ \\
SBR at screening & \\
$\quad$ Caudate nucleus & $1.21 \pm 0.30$ \\
$\quad$ Putamen & $1.18 \pm 0.28$ \\
SBR at 12-month f/u & \\
$\quad$ Caudate nucleus & $1.12 \pm 0.27$ \\
$\quad$ Putamen & $1.08 \pm 0.27$ \\
Annual percentage change (\%) & $-5.27 \pm 15.82$ \\
$\quad$ Caudate nucleus & $-7.18 \pm 12.48$ \\
$\quad$ Putamen & $391.3 \pm 53.5$ \\
\hline Interval between screening and 12-month f/u (days) \\
\hline Data are mean $\pm S D$ values. \\
f/u: follow-up, SBR: specific binding ratio.
\end{tabular}

lated region of the gene was associated with greater striatal DAT availability than 10 -repeats in normal subjects. ${ }^{16}$ The rs2652511 and re2937639 SNPs in the SLC6A3 gene were not associated with DAT availability. ${ }^{16}$ However, the rs 2652510 SNP with genotype GG in the SLC6A3 gene was associated with the risk of PD. ${ }^{17}$

In the present study we demonstrated that the mean SBRs of the caudate nucleus and putamen differed significantly between at screening and at the 12-month follow-up. The annual reduction rate was $7.18 \%$ in the putamen and $5.27 \%$ in the caudate nucleus, which is consistent with previous findings. ${ }^{7}$ No SNP significantly affected the decline of DAT availability in PD patients. However, association trends were observed in rs199347, rs356181, and rs3910105.

Nalls et al. ${ }^{18}$ found that SNP rs199347 on chromosome 7, which was observed in the GPNMB gene, was correlated with PD in Caucasians. They demonstrated that rs 199347 was associated with both methylation and expression changes in brain regions, and also showed that the risk allele (A) at rs 199347 on chromosome 7 was associated with an increased expression of NUPL2 as well as with decreased methylation of GPNMB in brain regions..$^{18}$ Those authors claimed that the risk at the locus containing rs 199347 might be due to increased transcription of NUPL2 and also be further bolstered by decreased methylation. Murthy et al. ${ }^{19}$ also reported that rs199347 with major allele AA increased the expression of GPNMB in the brain cortex and putamen in PD patients. Among human brain cell types, the expression of GPNMB was highest in oligodendrocytes, followed by microglia and astrocytes. ${ }^{19}$ A recent study demonstrated that GPNMB attenuates the astrocyte inflammatory response in rodents. ${ }^{20}$ Neuroinflammation mediated by activated glial cells is a common feature of $\mathrm{PD},{ }^{21}$ which suggests that the progression of $\mathrm{PD}$ via the activation of glial cells is attenuated by the increased expression of GPNMB through rs199347 with major allele AA. These results imply that the annual reduction rate
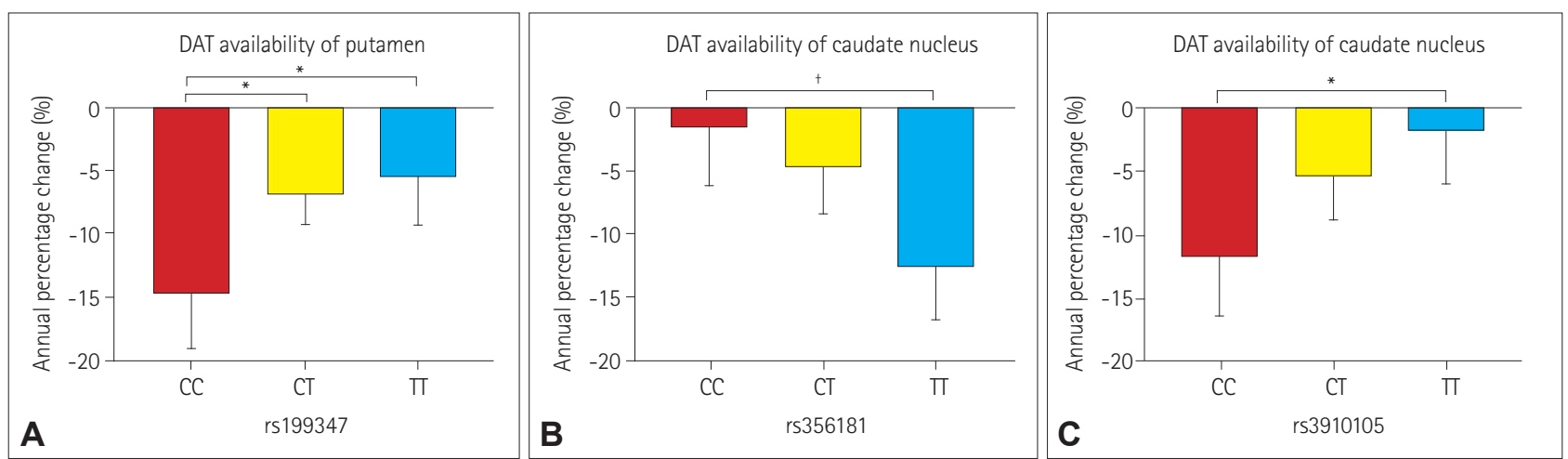

Fig. 2. Effects of single-nucleotide polymorphisms on the annual percentage change in DAT availability: rs199347 on the putamen (A), rs356181 on the caudate nucleus (B), and rs3910105 on the caudate nucleus (C) $\left({ }^{*} p<0.05,{ }^{+} p<0.01\right.$; data are means with $95 \%$ Cls). DAT: dopamine transporter. 
of DAT availability was reduced the most at allele CC and the least at allele AA. However, rs199347 was not associated with PD in a Taiwanese population ${ }^{22}$ or with PD in East Asians including residents of Singapore, Hong Kong, Malaysia, Korea, mainland China, and Taiwan. ${ }^{23}$ The present study utilized the PPMI database, the enrolled patients of various ethnicities, and so the correlation between changes in DAT availability and rs199347 might have been underestimated. A large population study of Caucasians should be performed to validate the protective effect of rs 199347 with allele AA of the GPNMB gene in the progression of PD.

SNPs rs356181 and rs3910105 on chromosome 4 were observed in the SNCA gene, which encodes $\alpha$-synuclein, ${ }^{24,25} \alpha-$ Synuclein consists of 140 amino acid residues and is implicated in the etiology of neurodegenerative diseases such as Alzheimer's disease, PD, dementia with Lewy bodies, and multiple-system atrophy. ${ }^{26} \alpha$-Synuclein is also considered to contribute to oligomers that are believed to play a key role in cell death and to be major components of Lewy bodies. ${ }^{26}$ Increased levels of SNCA mRNA have been reported in the midbrain and substantia nigra of PD patients compared with normal controls. ${ }^{27}$ However, a study evaluating the association between cerebrospinal fluid biomarkers including for a-synuclein and rs356181 and rs3910105 genotypes found no association between them. ${ }^{25}$

The SNCA gene has two CpG islands: 1) CpG-1 (located in the first exon) and 2) CpG-2 (located in the first intron). ${ }^{28}$ The methylation status of a $\mathrm{CpG}$ island is known to regulate SNCA in PD patients, and these islands are hypomethylated in PD patients compared with normal controls. ${ }^{28}$ However, another study found no correlation between hypomethylation of the SNCA-intron 1 and the $\alpha$-synuclein level. ${ }^{29}$ In contrast, the risk alleles of both rs356181 and rs3910105 were associated with greater DNA methylation of the $\mathrm{CpG}$ island of the SNCA gene..$^{30}$ Similarly, DNA methylation of the CpG island of the SNCA gene and SNPs rs356181 and rs3910105 appeared to be related with PD progression in the present study.

To the best of our knowledge, this is the first study to have investigated the correlation between genetic mutations and chronological changes in DAT availability. However, this study was subject to some limitations: 1) DAT availability could be affected by dopaminergic medications, 2) the reduction in striatal DAT availability might not be continuous, 3) the retrospective design makes selection bias inevitable, 4) the enrolled patients had various ethnicities that would affect the SNPs, and 5) the number of enrolled patients was small.

In conclusion, no SNP showed a statistically significant effect on the decline of DAT availability in PD patients. How- ever, we identified association trends: 1) rs199347 on the putamen, 2) rs356181 on the caudate nucleus, and 3) rs3910105 on the caudate nucleus. These findings indicate that the rs 199347, rs356181, and rs3910105 SNPs might affect the progression of PD.

\section{Conflicts of Interest}

The authors have no financial conflicts of interest.

\section{Acknowledgements}

This study was supported by Biomedical Research Institute Grant (2018 B007), Pusan National University Hospital.

PPMI-a public-private partnership-is funded by the Michael J. Fox Foundation for Parkinson's Research and funding partners, including abbVie, Avid, Biogen, Bristol-Myers Squibb, COVANCE, GE Healthcare, Genentech, GlaxoSmithKline, Lundbeck, Lilly, Merck, MesoScaleDiscovery, Pfizer, Piramal, Roche, Sanofi Genzyme, Servier, TEVA, and UCB.

All procedures performed in studies involving human participants were in accordance with the ethical standards of the institutional and/or national research committee and with the 1964 Helsinki declaration and its later amendments or comparable ethical standards.

\section{REFERENCES}

1. Lotharius J, Brundin P. Pathogenesis of Parkinson's disease: dopamine, vesicles and alpha-synuclein. Nat Rev Neurosci 2002;3:932-942.

2. Thomas AJ, Attems J, Colloby SJ, O’Brien JT, McKeith I, Walker R, et al. Autopsy validation of ${ }^{123} \mathrm{I}$-FP-CIT dopaminergic neuroimaging for the diagnosis of DLB. Neurology 2017;88:276-283.

3. Park E. A new era of clinical dopamine transporter imaging using ${ }^{123} \mathrm{I}-$ FP-CIT. J Nucl Med Technol 2012;40:222-228.

4. Djang DS, Janssen MJ, Bohnen N, Booij J, Henderson TA, Herholz K, et al. SNM practice guideline for dopamine transporter imaging with ${ }^{123}$ I-ioflupane SPECT 1.0. J Nucl Med 2012;53:154-163.

5. Suwijn SR, van Boheemen CJ, de Haan RJ, Tissingh G, Booij J, de Bie RM. The diagnostic accuracy of dopamine transporter SPECT imaging to detect nigrostriatal cell loss in patients with Parkinson's disease or clinically uncertain parkinsonism: a systematic review. EJNMMI Res 2015;5:12.

6. Benamer HT, Patterson J, Wyper DJ, Hadley DM, Macphee GJ, Grosset DG. Correlation of Parkinson's disease severity and duration with ${ }^{123}$ I-FP-CIT SPECT striatal uptake. Mov Disord 2000;15:692-698.

7. Kägi G, Bhatia KP, Tolosa E. The role of DAT-SPECT in movement disorders. J Neurol Neurosurg Psychiatry 2010;81:5-12.

8. Billingsley KJ, Bandres-Ciga S, Saez-Atienzar S, Singleton AB. Genetic risk factors in Parkinson's disease. Cell Tissue Res 2018;373:9-20.

9. McNeill A, Wu RM, Tzen KY, Aguiar PC, Arbelo JM, Barone P, et al. Dopaminergic neuronal imaging in genetic Parkinson's disease: insights into pathogenesis. PLoS One 2013;8:e69190.

10. Huertas I, Jesús S, García-Gómez FJ, Lojo JA, Bernal-Bernal I, Bonilla-Toribio $\mathrm{M}$, et al. Genetic factors influencing frontostriatal dysfunction and the development of dementia in Parkinson's disease. PLoS One 2017;12:e0175560.

11. Parkinson Progression Marker Initiative. The parkinson progression marker initiative (PPMI). Prog Neurobiol 2011;95:629-635.

12. García-Gómez FJ, García-Solís D, Luis-Simón FJ, Marín-Oyaga VA, Carrillo F, Mir P, et al. Elaboration of the SPM template for the standardization of SPECT images with ${ }^{123}$ I-Ioflupane. Rev Esp Med Nucl Imagen Mol 2013;32:350-356.

13. Tzourio-Mazoyer N, Landeau B, Papathanassiou D, Crivello F, Etard O, Delcroix N, et al. Automated anatomical labeling of activations in SPM using a macroscopic anatomical parcellation of the MNI MRI single-subject brain. Neuroimage 2002;15:273-289. 
14. Squire LR. Encyclopedia of Neuroscience. Boston: Elsevier Academic Press, 2009.

15. Fareed M, Afzal M. Single nucleotide polymorphism in genome-wide association of human population: a tool for broad spectrum service. Egypt J Med Hum Genet 2013;14:123-134.

16. van de Giessen E, de Win MM, Tanck MW, van den Brink W, Baas F, Booij J. Striatal dopamine transporter availability associated with polymorphisms in the dopamine transporter gene SLC6A3. J Nucl Med 2009;50:45-52.

17. Zhai D, Li S, Zhao Y, Lin Z. SLC6A3 is a risk factor for Parkinson's disease: a meta-analysis of sixteen years' studies. Neurosci Lett 2014;564: 99-104.

18. Nalls MA, Pankratz N, Lill CM, Do CB, Hernandez DG, Saad M, et al. Large-scale meta-analysis of genome-wide association data identifies six new risk loci for Parkinson's disease. Nat Genet 2014;46:989993.

19. Murthy MN, Blauwendraat C, Guelfi S, Hardy J, Lewis PA, Trabzuni D. Increased brain expression of GPNMB is associated with genome wide significant risk for Parkinson's disease on chromosome 7p15.3. Neurogenetics 2017;18:121-133.

20. Neal ML, Boyle AM, Budge KM, Safadi FF, Richardson JR. The glycoprotein GPNMB attenuates astrocyte inflammatory responses through the CD44 receptor. J Neuroinflammation 2018;15:73.

21. Wang Q, Liu Y, Zhou J. Neuroinflammation in Parkinson's disease and its potential as therapeutic target. Transl Neurodegener 2015;4:19.

22. Wu HC, Chen CM, Chen YC, Fung HC, Chang KH, Wu YR. DLG2, but not TMEM229B, GPNMB, and ITGA8 polymorphism, is associ- ated with Parkinson's disease in a Taiwanese population. Neurobiol Aging 2018;64:158.e1-158.e6

23. Foo JN, Tan LC, Irwan ID, Au WL, Low HQ, Prakash KM, et al. Genome-wide association study of Parkinson's disease in East Asians. Hum Mol Genet 2017;26:226-232.

24. Mata IF, Shi M, Agarwal P, Chung KA, Edwards KL, Factor SA, et al. SNCA variant associated with Parkinson disease and plasma alphasynuclein level. Arch Neurol 2010;67:1350-1356.

25. Kang JH, Mollenhauer B, Coffey CS, Toledo JB, Weintraub D, Galasko DR, et al. CSF biomarkers associated with disease heterogeneity in early Parkinson's disease: the Parkinson's Progression Markers Initiative study. Acta Neuropathol 2016;131:935-949.

26. Xu W, Tan L, Yu JT. Link between the SNCA gene and parkinsonism. Neurobiol Aging 2015;36:1505-1518

27. Tagliafierro L, Chiba-Falek O. Up-regulation of SNCA gene expression: implications to synucleinopathies. Neurogenetics 2016;17:145157.

28. Miranda-Morales E, Meier K, Sandoval-Carrillo A, Salas-Pacheco J, Vázquez-Cárdenas P, Arias-Carrión O. Implications of DNA methylation in Parkinson's disease. Front Mol Neurosci 2017;10:225.

29. Guhathakurta S, Evangelista BA, Ghosh S, Basu S, Kim YS. Hypomethylation of intron1 of $\alpha$-synuclein gene does not correlate with Parkinson's disease. Mol Brain 2017;10:6.

30. Hernandez DM. Genetic variation and DNA methylation in the context of neurological disease [dissertation]. London: University College London;2016. 